\title{
EDITORIAL
}

\section{Sociedad Chilena de Enfermedades Respiratorias: 75 años de historia}

\begin{abstract}
A fines de la década del 20, las cifras de morbilidad y mortalidad por Tuberculosis en Chile eran pavorosas y esta última era "una de las mayores del mundo". Hasta 1929 la práctica del tratamiento de estos pacientes "habia quedado en manos de algunos médicos que con heroicos sacrificios cuidaban a los tuberculosos y practicaban el neumotórax", introducido en Chile 15 años antes por el Dr. Manuel Moreno". En esa época "los hospitales generales evitaban recibir enfermos tuberculosos, y rara vez un paciente con esta enfermedad merecía la distinción de ser llevado a una clase magistral o detener por algunos minutos la atención del grupo que seguía al Profesor". El Dr. Sótero del Río estimaba que en 1935 esta enfermedad fue responsable de unas 15.000 muertes, con una tasa de 400 por 100.000 habitantes ${ }^{4}$. En ese año, él consideró que las causas respiratorias representaron un 36\% de la mortalidad general del país, con más de 41.000 fallecidos.
\end{abstract}

El Dr. Héctor Orrego Puelma había sido comisionado en 1928 para estudiar en Europa Farmacología y Terapéutica, ya que transcurridos cinco años de su egreso de la Escuela de Medicina, trabajaba como ayudante en la Cátedra respectiva. Estando en Francia ${ }^{3}$ y luego de un encuentro casual con el Dr. Hernán Alessandri, recibe de éste una invitación para asistir a la Clínica del Profesor Emile Sergent, quedando cautivado por este Maestro de la Medicina francesa. Se da cuenta de la gran importancia que adquiría una nueva rama de la Medicina, encargada de estudiar la Tuberculosis: la Tisiología. Aunque en la época era una enfermedad sin tratamiento específico, se habian logrado progresos en su conocimiento, y una notable disminución de la morbilidad y mortalidad utilizando medidas "directas e indirectas"4.

Regresa a Chile en 1929, y es nombrado Jefe de Sección del Hospital San José, por la Dirección General de Beneficiencia. A partir de este momento se inició una "revolución", que transforma este antiguo hospital, que recibía algunos enfermos tuberculosos, en un Servicio a la usanza de los que había observado en Europa, entusiasmando a otros médicos en la especialización en Tisiología. En poco tiempo logró que fuera dedicado exclusivamente a esta enfermedad, transformando entonces el antiguo lazareto en un campo clínico y una enfermedad hasta entonces despreciada por los médicos en una fuente de ciencia e investigación.

En forma paralela el Dr. Sótero del Río se encontraba también en Europa, estudiando Anatomía Patológica en la Universidad de Strasburgo, donde adquiere también conocimientos en tisiología ${ }^{3}$. A su regreso a Chile en 1929, se hace cargo de la Jefatura de Tisiología de la Dirección General de Beneficiencia lo cual le permite unir sus esfuerzos al Dr. Orrego, su ex-compañero de curso, en la creación en Chile de la nueva especialidad.

A partir de este momento, en que se unen a su regreso a Chile quienes han sido considerados con justicia los padres de la Especialidad en nuestro pais ${ }^{3}$, se funda la Sociedad Chilena de Tisiología, el 13 de Marzo de 1930. Su primer directorio estuvo formado por los Drs. Héctor Orrego, Sótero del Río, Armando Alonso, Rolando Castañón y Manuel Sánchez. También participaron en la reunión fundacional los Drs. Fernando Cruz, Félix Bulnes, Arturo Espina, Max Vega, Salomón Margullis y Gonzalo Corbalán.

En forma simultánea el Dr. Orrego inicia cursos de formación para médicos, y se crea la Cátedra de Tisiología con su nombramiento como Profesor Extraordinario primero, y en 1934 como Profesor Titular. Mientras desarrolla una fructífera labor asistencial y académica, formando los médicos que luego se harán cargo de los pacientes en diversos lugares del país, promueve también desde el inicio en la Sociedad de Tisiología, la necesidad de contar con estudios de diferentes aspectos de la enfermedad, y un número de camas suficientes para un adecuado tratamiento de la Tuberculosis. En los años siguientes ambos objetivos se cumplen plenamente, ya que la Sociedad mantiene reuniones periódicas, con presentación de unos 40 trabajos clínicos al año, y por otra parte comienza la construcción de modernos sanatorios en las cercanías de Santiago ${ }^{4}$ (El Peral, actual Sótero del Rio ${ }^{6}$ ), en Valparaíso y Putaendo.

El Dr. Sótero del Río fue nombrado Jefe de la Sección Broncopulmonares del Hospital Salvador, y se hace también cargo de la Sociedad Constructora de Establecimientos Hospitalarios. En 1935 
fue fundada la revista Aparato Respiratorio y Tuberculosis, y en el mismo año el Dr. Sótero del Río asume el cargo de Director General del Servicio de Beneficiencia y Asistencia Social y poco después, en 1937, renuncia a la Jefatura en el Hospital del Salvador, para dedicarse de lleno a una exitosa carrera política, que le permitiría contribuir en forma decisiva al progreso de la Especialidad, y especialmente al bienestar de los pacientes. El concurso por su cargo es ganado por el Dr. Héctor Orrego, quien traslada entonces la Cátedra de Tisiología a este hospital.

Otro aspecto primordial en el desarrollo de la Medicina chilena en esa época lo constituyó la creación de la ley de Medicina Preventiva, uno de los enormes aportes del Dr. Eduardo Cruz Coke L. Esta ley, revolucionaria en su época, promovía el examen periódico y sistemático para la detección precoz de las enfermedades, entre ellas la Tuberculosis, como lo señaló el propio autor en una sesión de la Sociedad de Tisiología ${ }^{5}$, pero además extendía los controles a los familiares, y consideraba un aporte económico que facilitara la recuperación del enfermo.

Este primer periodo de la Sociedad culmina con la introducción, a fines de la década del 40 de la estreptomicina, primer antibiótico que lograba la curación de la Tuberculosis. Posteriormente la introducción del ácido para aminosalicílico y de la "maravillosa realidad de la hidrazida del ácido isonicotínico en 1952"' contribuyeron al brusco descenso de la mortalidad por Tuberculosis, que en pocos años pasó de 228 a 109 por 100.000, para continuar descendiendo con rapidez en los años posteriores.

El siguiente periodo se caracteriza por la introducción de estudios funcionales, y la mayor importancia relativa de otras enfermedades respiratorias al disminuir la tuberculosis. En forma paralela, se produjo el despegue de la cirugía torácica, que aunque habia presentado grandes progresos desde la década de los 40, planteando soluciones quirúrgicas para enfermos tuberculosos, como fue la introducción de la toracoplastía.

Es a partir de 1950 que los indices de morbimortalidad quirúrgica comienzan a disminuir considerablemente, de la mano del inicio de la evaluación funcional pulmonar ${ }^{6}$. Como suelen ser las novedades en Medicina, algunos se entusiasmaron con estos procedimientos, y muchos resistieron. El Dr. E. Motles, a su regreso de EE.UU. instaló el primer laboratorio de función pulmonar en el Sanatorio El Peral, y desde 1947 presentó trabajos en la Sociedad analizando el impacto de diferentes patologías médicas, así como distintas terapias quirúrgicas (toracoplastía, frenoparálisis, lobectomías, etc). Poco después comenzaron los estudios funcionales en el Hospital de la Universidad Católica (Dr. E. Cruz), el Hospital del Salvador (Dr. H. Donoso) y el recientemente inaugurado Hospital del Tórax (Dr. E. García Campos) $)^{3}$. El entusiasmo de los médicos jóvenes por estas nuevas herramientas permitió comprender con mayor precisión las enfermedades que afectan el aparato respiratorio, y aprovechar el gran rendimiento clínico que estas técnicas poseen. En esta época comienzan también los estudios más acabados de las enfermedades profesionales ${ }^{6}$, especialmente la silicosis pulmonar, que tuvieron su gran promotor en el Dr. Hernán Oyanguren.

La inauguración en 1954 de nuevos establecimientos, como el Hospital del Tórax - "edificio moderno y diseñado para llevar el desarrollo de la especialidad a nuevos horizontes "', y el Hospital San Juan de Dios, fueron hitos en la modernización de la Tisiología en su tránsito a la Neumotisiología. En el primer establecimiento se instala la Cátedra del Profesor Héctor Orrego Puelma, y en al segundo se traslada la Cátedra E. de Medicina del Profesor Rodolfo Armas Cruz.

En esta etapa la Sociedad cambia de nombre, siguiendo la dinámica que le imponen los nuevos conocimientos: Sociedad Chilena de Tisiología y Enfermedades Broncopulmonares" (1951), y luego Sociedad Chilena de Enfermedades del Tórax y Tuberculosis" (1956) ${ }^{3}$. El Hospital Clínico de la Universidad Católica es pionero en la integración de la especialidad con la Medicina Interna, y destaca especialmente por el enfoque fisiopatológico de las enfermedades respiratorias. En el Hospital San Juan de Dios, el Servicio de Medicina, que gracias a la brillante conducción del Profesor Armas alcanzará rápidamente el sitial histórico que conocemos, recibe como becados a sus mejores ex alumnos, iniciando el desarrollo de las subespecialidades. Entre ellos, el Dr. Victorino Farga ${ }^{3}$ toma conciencia de la enorme prevalencia de la patología respiratoria y la falta de preocupación por ella. Como consecuencia viaja a formarse a Filadelfia, regresando en 1957 para desarrollar la especialidad. Es seguido un año después por el Dr. Edgardo Carrasco, quien se especializó en Fisiopatología y Alergia respiratoria, en Boston, iniciando a su regreso una pionera y fructífera labor, incentivando el interés por esta nueva disciplina.

En el siguiente periodo se produce la consolidación de la especialidad en su concepción actual. Es claramente la continuación de la anterior, y en ella aparecen ya los estudios más específicos tanto de fisiopatología como de Alergia respiratoria. De igual manera, asistimos a los albores de la especialidad en pediatría, tema que se desarrollará en otro artículo, por la extensión e importancia que tiene. 
En esta época comienzan los estudios de campo en tuberculosis, investigando de manera cientifica y operacional la eficacia de diferentes esquemas, que permiten llevar el control del problema de la tuberculosis a terrenos no sospechados previamente, acortando la duración de la terapia y disminuyendo la tasa de abandonos. El pionero en este campo fue el Profesor Victorino Farga, quien se trasladó al Hospital del Tórax en 1966, al ganar por concurso la Cátedra de Tisiología, que había dejado el Profesor Orrego al jubilar. A finales de esta década presenciamos el nacimiento de las Unidades de Cuidados Intensivos Respiratorios, primero en el Hospital Clínico de la Universidad Católica y unos años después en el Hospital del Tórax.

El Dr. Carrasco permanece a cargo del Centro de Enfermedades Broncopulmonares del Hospital San Juan de Dios, creando una escuela rigurosa en que inician su formación muchos médicos jóvenes ${ }^{3}$. En 1974 el Hospital del Tórax se transforma en Instituto de Enfermedades Respiratorias y Cirugía Torácica, y asume como su director el Dr. Edgardo Carrasco, quien era Profesor Titular de Medicina. Desde este instituto continúa su labor docente y crea en 1985 la revista Enfermedades Respiratorias y Cirugía Torácica, que se transforma en 1987 en el órgano oficial de nuestra Sociedad. En 1990 cambia de nombre, a la actual Revista Chilena de Enfermedades Respiratorias ${ }^{7}$.

En las últimas décadas a medida que se solucionan viejos problemas de salud, aparecen nuevos desafios: los efectos de la contaminación atmosférica ${ }^{8}$ en la salud de la población y las consecuencias del tabaquismo lideran las preocupaciones.

Actualmente nos enfrentamos a estos nuevos desafios. En Chile, las enfermedades infecciosas respiratorias constituyen la segunda causa de pérdida de años de vida saludable en el país, y el Asma Bronquial la séptima9. Las consecuencias del tabaquismo son la mayor carga de enfermedad en nuestro país, y las cifras actuales demuestran que un $42 \%$ de la población es fumadora y un $22 \%$ de los chilenos tiene síntomas respiratorios crónicos ${ }^{10}$. Mientras en la lucha antituberculosa hemos alcanzado el umbral de la fase de eliminación de la enfermedad, y en los últimos años hemos asistido a una disminución de la mortalidad por infecciones respiratorias bajas, es probable que presenciemos en la próxima década un aumento de las enfermedades crónicas no transmisibles, especialmente Cáncer Pulmonar y EPOC.

Las profundas transformaciones demográficas y sanitarias ocurridas en nuestro país han modificado las causas de morbilidad y mortalidad. Nuestra Sociedad ha participado durante 75 años en la prevención y tratamiento de las enfermedades respiratorias, y se encuentra preparada para continuar haciéndolo ${ }^{11}$. Para ello la Comisión Científica se encuentra elaborando el reglamento para nuevas becas de perfeccionamiento, han sido creados fondos concursables para investigación en temas de ciencias biomédicas, clínica y epidemiología y se han establecido estímulos para la elaboración de proyectos multicéntricos. Por otra parte, en los últimos tiempos nuestra Sociedad ha tenido una activa participación en la elaboración de Guías Clínicas, en conjunto con el Ministerio de Salud, y su Comisión Tabaco o Salud ha liderado la participación de las Sociedades Científicas en promover la ratificación en el Congreso del Convenio Marco para el Control del Tabaco.

\section{Dr. Manuel Barros Monge Presidente Sociedad Chilena de Enfermedades Respiratorias Profesor Adjunto, Escuela de Medicina, Universidad de Valparaíso}

\section{BIBLIOGRAFÍA}

1 .- GARCÍA V R. Crónica. Aparato Respiratorio y Tuberculosis 1953; 18: 315-20.

2 .- ORREGO H. Apuntes sobre la historia de la Tuberculosis. Aparato Respiratorio y Tuberculosis 1957; 18: 61-77.

3.- MENA G. El tránsito de la Tisiología a la especialidad actual en Chile. Enferm Respir Cir Torác 1989: 5; 99-108.

4.- DEL RÍO G S. La lucha antituberculosa. Aparato Respiratorio y Tuberculosis 1937; 2: 84-94.

5.- CRUZ COKE E. Medicina preventiva en relación con tuberculosis. Aparato Respiratorio y Tuberculosis 1938; 3: 113.

6.- MOTLES E. Evolución de los conocimientos y conceptos fisiológicos, patológicos y terapéuticos en relación al aparato respiratorio en los últimos 50 años. Enf Respir Cir Torác 1990: 6; 91-7.

7.- OYARZÚN M. Evolución de las revistas sobre enfermedades respiratorias y tuberculosis en Chile. Rev Chilena Enf Respir 1996; 12: 103-16.

8.- OYARZÚN G M. Ambiente y salud en Chile, relación presente-futuro. Rev Chilena Enf Respir 1998; 14: 30-9.

9.- Ministerio de Salud. Estudio de Carga de Enfermedad 1993. Informe No 4. Resumen Ejecutivo. www.minsal.cl

10.- Encuesta Nacional de Salud 2003. Ministerio de Salud. www.minsal.cl

11.- BARROS M. Desafíos y oportunidades de la Sociedad Chilena de Enfermedades Respiratorias. Rev Chil Enf Respir 2004; 20: 7-8. 
